\title{
Hidden linear defects in surfactant onions revealed by coalescence into lamellar layers
}

\author{
Yoshimasa Izaki $\odot,{ }^{1}$ Rei Kurita, ${ }^{1,2}$ and Hajime Tanaka $\odot^{1,3, *}$ \\ ${ }^{1}$ Department of Fundamental Engineering, Institute of Industrial Science, University of Tokyo, 4-6-1 Komaba, \\ Meguro-ku, Tokyo 153-8505, Japan \\ ${ }^{2}$ Department of Physics, Tokyo Metropolitan University, 1-1 Minamioosawa, Hachiouji-shi, Tokyo 192-0397, Japan \\ ${ }^{3}$ Research Center for Advanced Science and Technology, University of Tokyo, 4-6-1 Komaba, Meguro-ku, Tokyo 153-8505, Japan
}

(Received 17 May 2021; accepted 1 October 2021; published 1 November 2021)

\begin{abstract}
The onion-like multilamellar structure is ubiquitous in various soft materials, such as surfactant solutions, biological membranes, and block copolymers, as well as hard materials such as carbon. Onions appear to be perfectly spherical, giving the impression that it has a point-symmetric, seamless, and defect-free internal structure. Here, we focus on surfactant onions formed in the sponge phase and experimentally study whether the interior of surfactant onions is defect-free and isolated from the surrounding matrix or not. By directly observing the coalescence process between onions and a planar lamellar domain with optical microscopy, we have demonstrated that the onions do not have a completely closed structure but have a linear array of defects along the radial direction formed upon onion formation. In other words, the onion structure is symmetric axially but not spherically, like the plant-onion structure. This discovery of the hidden breakdown of spherical symmetry sheds fresh light on the onion's internal topology and formation mechanism.
\end{abstract}

DOI: 10.1103/PhysRevResearch.3.043094

\section{INTRODUCTION}

Amphiphilic molecules such as surfactants are known to spontaneously form various types of self-organized structures in aqueous solutions, such as the lamellar (lyotropic smectic), onion (or multilamellar vesicle), and sponge structures [1]. Multilamellar structures are also an essential organization of biological bilayer membranes, i.e., lipid-protein complexes [2,3]. For example, the onion-like topology of double-layer mitochondrial inner membranes has been known to form instead of cristae organization when membranes cannot bend sharply, e.g., in the absence of ATP synthase dimerization [4-6]. Multilamellar vesicles are also important for various applications, such as drug delivery [7,8] and microreactors $[9,10]$. Furthermore, the formation of onion-like structures is not limited to soft materials and can be seen in monoatomic systems such as carbon [11-13], which have attracted a renewed interest in this organization's intriguing structure.

The onion-like multilamellar vesicle has long been known to be formed from the lamellar phase of lipid or surfactant systems either spontaneously or by shaking them [14-17]. Later, it was found that the onion structure is formed from the lamellar phase of surfactant solutions by shear-induced mechanical instability [18]. Such shear-induced onion formation can occur directly from the planar lamellar phase or through

\footnotetext{
*tanaka@iis.u-tokyo.ac.jp

Published by the American Physical Society under the terms of the Creative Commons Attribution 4.0 International license. Further distribution of this work must maintain attribution to the author(s) and the published article's title, journal citation, and DOI.
}

an intermediate phase (cylindrical onions or leeks) [19,20]. However, the formation mechanism of onions by shearing the lamellar phase of surfactant solutions has remained a long-standing mystery in soft matter science, despite extensive experimental [21-31], theoretical [32-36], and numerical studies [37]. The onion size $R$ decreases with an increase in the shear rate $\dot{\gamma}$ as $R \propto \dot{\gamma}^{-1 / 3}$ when onions are formed from a lamellar phase. On the other hand, it was shown that $R \propto \dot{\gamma}^{-1}$ when onions are formed from a phase-separating lamellar-sponge mixture (see, e.g., Ref. [38]).

Later, we found another route of onion formation via spontaneous nucleation of a lamellar domain from the isotropic sponge phase [39]. We revealed the sponge-to-onion transformation pathway by direct optical-microscopy observation of the transformation process. This thermodynamic route is free from mechanical perturbation such as shear. The onion formation from a micellar phase was also reported [40,41]. We point out that onions can be formed in not only surfactant solutions but also other amphiphilic systems such as block copolymers [42-46] and Janus dendrimers [47]. Recently the sponge-to-lamellar transition, including onion formation, has also been discovered for Janus dendrimers [48], indicating the ubiquity of such spontaneous onion formation from the sponge phase.

These onions are often regarded as spherical multilamellar vesicles whose inside is entirely isolated from the outside. Onions locally have lamellar (or smectic) order but are topologically distinct from the planar lamellar phase. Thus, the transformation between onions and planar lamellar domains must involve a topological change of membrane organization. Based on the closed nature of the onion structures, applications of onions to drug delivery are proposed. However, when onions spontaneously form from the sponge phase, they 
coexist with the sponge phase, an isotropic bicontinuous phase characterized by the negative Gaussian curvature. Since the energy cost forming edges of bilayer surfactant membranes far exceeds the thermal energy, it is considered that surfactant membranes do not have any edges, and thus, are seamlessly connected [49]. It suggests a possibility that the onion structure intrinsically has topological defects. Since topological defects may provide a material transport path between the inside and outside of an onion, it should crucially affect the transport functions, e.g., drug delivery. Thus, the critical question is whether onions have perfect spherical symmetry and are made of seamless membranes [see Fig. 1(a)] or they have topological defects as a consequence of the sponge-toonion structural transformation [see Fig. 1(b)], i.e., whether the onion structure is closed or open. We address this fundamental question by directly observing a coalescence process between an onion and a planar lamellar domain with optical microscopy. We will show that the onion does not have a seamless structure with spherical symmetry but has a linear arrangement of topological defects created upon its formation.

\section{EXPERIMENTAL METHODS}

To address whether the onion structure is seamless or not [see Figs. 1(a) and 1(b)], we study the dynamic process of collision and coalescence of an onion to a planar lamellar domain.

\section{A. Samples and the phase diagram}

The surfactant molecules we used were nonionic surfactants, $\mathrm{C}_{10} \mathrm{E}_{3}$ (tri-ethylene glycol mono n-decyl ether, Nikko Chemicals Inc., BD-3SY). When we dissolve the surfactant molecules into water, they spontaneously form bilayer membranes in water in a specific region of the phase diagram. The bilayer membranes further form a higher-order membrane organization, and thus, this system is also called lyotropic liquid crystal (LC). At a low temperature, membranes form a lamellar phase $\left(L_{\alpha}\right.$, smectic LC) with a one-dimensional periodic order of surfactant bilayers. On the other hand, at a high temperature, they form a sponge phase $\left(L_{3}\right.$, isotropic fluid), which is a random bicontinuous structure of the membranes and water [1]. There is a coexistence region of the two phases between these two homogeneous phases, reflecting the first-order nature of the transition between the sponge and lamellar phases. The boundaries between the coexistence region and the two phases monotonically increase with an increase in the surfactant concentration $\phi$. The phase diagram of the $\mathrm{C}_{10} \mathrm{E}_{3}$ /water mixture is shown in Ref. [50] (see also Ref. [25]).

A lamellar phase of these systems is known to be stabilized by Helfrich interactions (entropic repulsions) [51]. In the hyperswollen regime, the intermembrane spacing $d$ in the lamellar structure is inversely proportional to the composition $\phi$ (we note that there is a logarithmic correction to the relation, characteristic of two-dimensional fluctuation systems [1]); for example, it is $0.014 \mu \mathrm{m}$ for $20.0 \mathrm{wt} \%$ and $0.18 \mu \mathrm{m}$ for 1.5 wt $\%$. Figure 1(c) shows the phase diagram of $\mathrm{C}_{10} \mathrm{E}_{3} / \mathrm{H}_{2} \mathrm{O}$ mixtures [25], constructed based on optical microscopy observation. Since the phase transition between the lamellar and


FIG. 1. Possible internal structures of onions and phase diagram. (a) Schematic picture of the onion's internal structure with perfectly spherical symmetry. $I$ and $O$ represent the inside and outside water regions of the sponge phase, respectively. Note that $I$ and $O$ are only distinguished by symmetry, but both are mainly made of water and have identical components. (b) Schematic picture of the onion's internal structure with defects. The dashed line represents a linear arrangement of defects whose details are not explicitly shown because of the lack of microscopic understanding of the defect structure. It is considered that there should be no edges of membranes [49], and thus, the inside-outside distinction should be preserved strictly. (c) The phase diagram of $\mathrm{C}_{10} \mathrm{E}_{3} / \mathrm{H}_{2} \mathrm{O}$ mixtures. Note that it is shown in a log-linear plot. In the coexistence region, the volume fractions ( $v_{\alpha}$ for $\mathrm{L}_{\alpha}$ and $v_{3}$ for $\mathrm{L}_{3}$ ) are determined from the phase diagram, following the lever rule. With an increase in temperature, the volume fraction changes monotonically from $v_{3}=0$ (i.e., $v_{\alpha}=1$ ) in the onephase lamellar region (at a low temperature) to $v_{3}=1$ (i.e., $v_{\alpha}=0$ ) in the one-phase sponge region (at a high temperature) through the coexistence region (where $v_{3}+v_{\alpha}=1$ ). Schematic drawings of the lamellar and sponge structures are also shown.

sponge phases is weakly first-order, a new phase is formed via a nucleation process.

For most cases, we confined a sample between two parallel cover glasses whose spacing is approximately $190 \mu \mathrm{m}$. Then, we heated the sample to prepare the homogeneous sponge phase first and then cooled it rapidly at $-10.0 \mathrm{~K} / \mathrm{min}$ into the coexistence region.

\section{B. Temperature quench protocols}

In a sample confined between two parallel cover glasses in the cooling process, the lamellar phase is first nucleated heterogeneously on the sample cell surfaces by surface wetting effects. Then, homogeneous nucleation occurs in the sponge phase far from the sample cell surfaces [52]. We quenched a sample deeper than the difference in the minimum quench depth, $\Delta T$, between heterogeneous and homogeneous nucleation for observing both the heterogeneous and homogeneous nucleation processes. Since $\Delta T$ increases with the surfactant concentration $\phi$, the quench depth is shallow for a low concentration (e.g., $\Delta T \sim 0.1 \mathrm{~K}$ at $3 \mathrm{wt} \%$ ) whereas deep for a high concentration (e.g., $\Delta T \sim 1 \mathrm{~K}$ at $20 \mathrm{wt} \%$ ). In our experiments, we chose the cooling rate, and the sample thickness (mainly about $200 \mu \mathrm{m}$ ) [52]. We confirmed that the homogeneous nucleation in the sample cell's middle is not influenced by the heterogeneous nucleation on the sample cell's walls. 


\section{Characteristic features of this system}

This system is characterized by a large intermembrane spacing $d$ since the lamellar phase can be formed in a dilute regime of the phase diagram (e.g., about $0.1 \mu \mathrm{m}$ for the concentration $\phi=3 \mathrm{wt} \%$, which we can measure by the Bragg reflection of light; see, e.g., Ref. [53]). This large length scale enables us to "directly" observe the initial process of nucleation and growth of the lamellar phase with optical microscopy [39]. The microscopy resolution is high enough to observe small lamellar nuclei just after their formation. Furthermore, the large characteristic timescale (approximately proportional to $d^{3}$ ) [54,55] makes the dynamic process of nucleation and growth of the lamellar phase slow enough for us to follow in real time.

We cooled a sample from the one-phase sponge region to the lamellar-sponge coexisting region to observe the lamellar phase's homogeneous nucleation [see Fig. 1(c)]. A typical process of the onion formation from the sponge phase via lamellar nuclei is shown in Fig. 2(a).

\section{RESULTS AND DISCUSSION}

\section{A. Onion formation and floatation}

For $\phi=2-10 \mathrm{wt} \%$, the nucleation rate is low for a shallow quench. Therefore, we can observe the nucleation-growth process of an individual nucleus without interference from other nuclei (no overlap of depletion layers). The process of onion formation is summarized as follows [39]. After a temperature quench, the lamellar phase is spontaneously nucleated in bulk as spindles. The observation of the nuclei of various orientations tells us that the spindle-like lamellar domain has a lens shape [Fig. 2(a1)]. When a lens-shaped nucleus grows to a certain size, it starts to bend [Fig. 2(a2)] and changes its shape from lens to sphere [Fig. 2(a1)-2(a6)]. It is because the interfacial energy penalty for the lens shape exceeds the bending energy cost. This shape transformation proceeds while keeping axisymmetry: During the transformation, the edge of a lens-shaped nucleus remains circular, and the curved nucleus has "cup-like" geometry (see the nucleus in Fig. 2(a3), whose axisymmetric axis is on the image plane). Finally, the sponge phase remaining around the center [Fig. 2(a6)] gradually transforms into the lamellar phase, and thus, the nucleus eventually forms a spherical lamellar domain, i.e., an onion [see Fig. 2(b)].

As shown in Ref. [52], a planar lamellar domain is formed by wetting-induced heterogeneous nucleation on the flat glass walls of the sample cell immediately after a temperature quench, i.e., before onions are formed. This lamellar (smectic) phase is homeotropically aligned to a glass wall. Although we did not measure the thickness of the planar lamellar layer, it may be several to ten microns, according to our previous paper [52]. Onions are formed by homogeneous nucleation in bulk after the planar lamellar layer is formed by heterogeneous nucleation. These onions are lighter than the sponge phase since surfactant bilayer membranes have a lower density than water, and onions have a higher concentration of surfactants than the sponge phase [see Fig. 1(c)]. Therefore, the onions float to the top, as shown in Fig. 2(b).
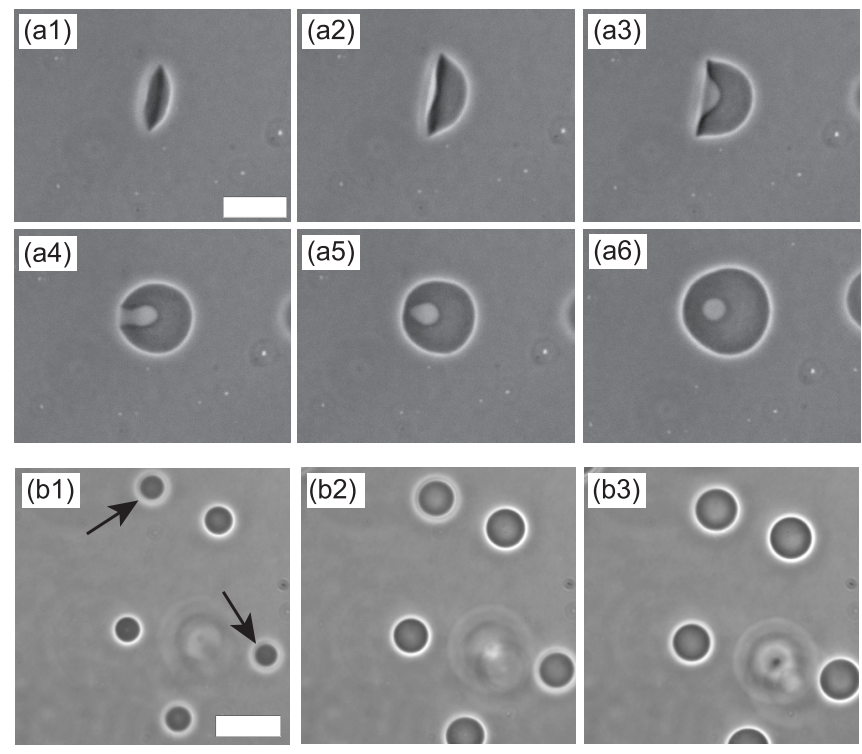

FIG. 2. Onion formation and floatation. (a) The process of onion formation. Here $\phi=3.0 \mathrm{wt} \%$ and $T=31.0{ }^{\circ} \mathrm{C}$. Images [(a1)-(a6)] correspond to $10 \mathrm{~s}, 100 \mathrm{~s}, 180 \mathrm{~s}, 240 \mathrm{~s}, 300 \mathrm{~s}$, and $450 \mathrm{~s}$, respectively, after a temperature quench from the one-phase sponge region to the sponge-lamellar coexisting region. First, lens-shaped lamellar nuclei are formed, but they gradually change from lens to spherical. The shape transformation takes place because the surface energy exceeds the elastic deformation energy. The onion is not perfectly spherical in this relatively short time, and a sponge region remains around its center. However, it eventually becomes an almost perfectly spherical shape, and the sponge region in the middle also almost wholly transforms to a lamellar structure (see, e.g., Figs. 2(b) and 2(e) in Ref. [39], where we can see a perfectly spherical shape and a polarizing microscopy image consistent with the spherical structure with smectic order, respectively). We can also see spherical onions in panel (b) [note that the transformation kinetics is much faster for $\phi=5.0 \mathrm{wt} \%$ than $3.0 \mathrm{wt} \%$ (the characteristic time scale is roughly proportional to $\phi^{-3}$; see the main text)]. (b) The process of slow floatation of onions towards the top cover glass whose surface is covered by the flat lamellar phase. Here $\phi=5.0 \mathrm{wt} \%$ and $T=31.0^{\circ} \mathrm{C}$. Images (b1)-(b3) correspond to $100 \mathrm{~s}, 600 \mathrm{~s}$, and $800 \mathrm{~s}$, respectively, after a temperature jump. As time goes on, we can see that onions pointed by arrows are approaching the top glass wall, on which the microscope is focused. We note that the lamellar phase is lighter than the sponge phase since the surfactant's density is lower than water. Thus, the buoyancy force is acting on onions. The scale bars correspond to $200 \mu \mathrm{m}$ for both.

\section{B. Deformation and molting of onions induced by collision to the planar lamellar layer}

The critical question here is whether the onion structure formed in the process described above has a perfect seamless closed interior structure as depicted in Fig. 1(a) or has defects as depicted in Fig. 1(b). We answer this question by examining an onion's collision and coalescence process to a planar lamellar domain.

Here we show the dynamic process of the collision and coalescence of onions and the planar lamellar layer formed on the top glass wall in Figs. 3 and 4 (see also Movie S1 in the Supplemental Material [56]). Now we infer what happens 

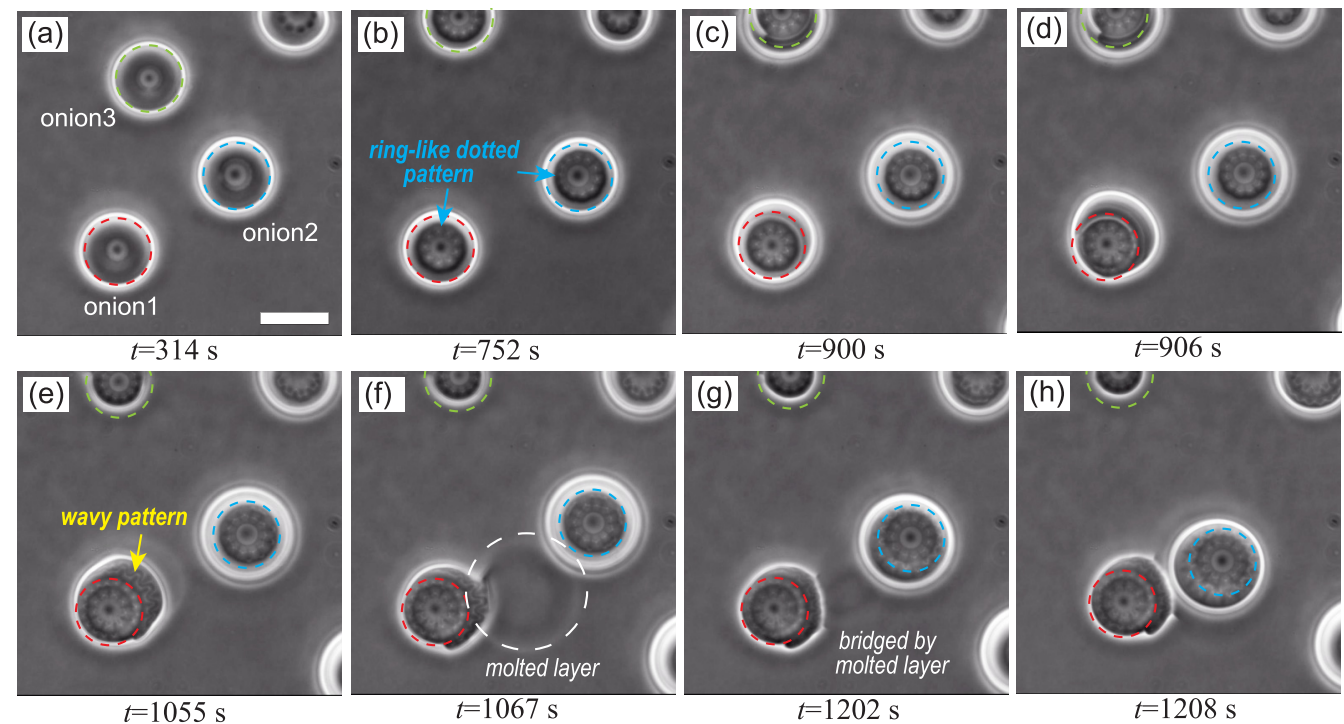

FIG. 3. Coalescence process between an onion and a flat lamellar domain formed on the cover glass. The sample of $10.0 \mathrm{wt} \% \mathrm{C}_{10} \mathrm{E}_{3} \mathrm{was}$ quenched from 33.7 to $32.7{ }^{\circ} \mathrm{C}$. The scale bar corresponds to $200 \mu \mathrm{m}$. The time is measured after the quench (see also Movie S1 in the Supplemental Material [56] for the dynamic process, in which the acceleration factor is $\times 150$ ). The size of the onion just after contact [see (a)] is shown by red, blue, green dashed circles with the same radius for onions 1, 2, and 3, respectively, for [(a)-(h)]. We always set the circle center at the central dark spot on an onion. We can see the elementary process of merging the onion to the flat lamellar layer in the form of molting. The dark circular region appears in the center after an onion contacts the planar lamellar layer (a). Then, the onion exhibits mechanical instability to create a ring-like white dotted pattern (b). This instability may be induced by the deformation of onions pressed to the top glass wall by buoyancy and adhesion forces [see Fig. 2(b)]. In this process, the onion's surface wets the planner lamellar layer formed on the glass wall, leading to a gradual increase in the contact area. This induces the onion deformation from a spherical shape, which may induce the instability forming a ring-like dotted structure (see the main text). The shape deformation from a sphere [see Fig. 4(i)] also leads to the molting of the outer shell of the onion. Then, the molted part wets the planar lamellar layer [see (e)- $(\mathrm{g})]$. We can see the molted layer spreads mainly on its right-hand side for onion 1 [see the region surrounded by the white dashed circle in (f)], which bridges onions 1 and 2 via the molted layer (g) and leads to their collision (h). For onion 2, the molted layer is spread almost isotropically around it. For onion 3, the molted layer is probably formed in its upper side (outside the image), and the remaining core part becomes smaller than the initial size (see green dashed circles). This spreading-type difference may be induced by the difference in the direction of a linear array of defects. If the direction is normal to the planar lamellar layer, the spreading is isotropic; otherwise, anisotropic.

in this process. When onions contact the planar lamellar domain formed on the top cover glass's inner surface, they are deformed by the buoyancy and adhesive forces to a nonspherical shape with a flat top. We can detect the onset time of this flattening from Movie S1 in the Supplemental Material [56]. In our microscopy observation, the focus is located near a scratch on the cover glass's inner surface. Thus, we can determine the contact time of an onion to the planar lamellar phase formed on the glass's inner surface from the emergence of a dark circular region in Movie S1 in the Supplemental Material [56] [see also Figs. 3(a) and 4(a)]. For $\phi=3.0 \mathrm{wt} \%$ at $T=31.0^{\circ} \mathrm{C}$, the contact time is about $t=310 \mathrm{~s}$.

The contact of the onion to the planar lamellar layer leads to flattening the contacted part of the onion, i.e., the deviation from a spherical shape. The resulting increase of the onion surface area stretches membranes in the outer part of the onion, creating tension [see the light blue color arrows in Fig. 4(i)]. Wetting-induced adhesion further increases the contact area with time [see Figs. 3 and 4], accompanying the more substantial shape deformation of the onion. Once the accumulated tension on the outer shell of the onion exceeds a critical value, the linear defect array in the onion formed in its formation process is forced to open up [see Figs. 4(i) and 5], and the surface part of the onion is detached from the core part of the onion and spreads on the planar lamellar layer [see Figs. 3(e), 3(f), and 4(f)-4(h)]. This process is repeated, and the onion is eventually wholly absorbed into the planar lamellar phase, which is a lower free energy state than the metastable onion phase.

The way of molting seems to depend on the condition. The molted layer spreads mainly on its right-hand side for onion 1 [see the white dashed circle in Fig. 3(f)], which bridges onions 1 and 2 via the molted layer [Fig. 3(g)] and leads to their collision [Fig. 3(h)]. On the other hand, for onion 2, the molted layer is spread almost isotropically around it. For onion 3, the molted layer is moved to its upper side (outside the image), and the remaining core part becomes smaller than the initial size [see green dashed circles in Figs. 3(e)-(h)]. This spreading-type difference may be induced by the difference in the direction of a linear array of defects. If the direction is normal to the planar lamellar layer, the spreading is isotropic; otherwise, anisotropic.

Now, we turn our attention to the ring-like dotted pattern observed in the onion contacting the planar lamellar layer (see Figs. 3 and 4). The edge of the contacted region should have a sharp change in the curvature compared to the constant 


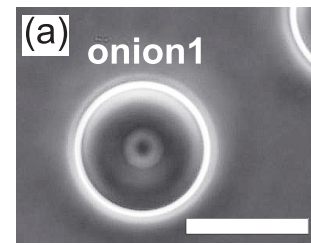

$t=310 \mathrm{~s}$



$t=910 \mathrm{~s}$

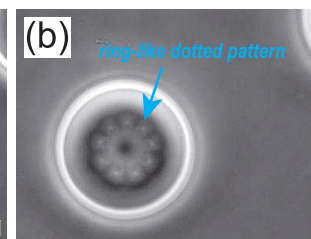

$t=470 \mathrm{~s}$

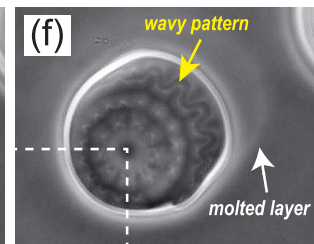

$t=1055 \mathrm{~s}$

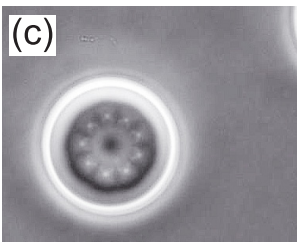

$t=760 \mathrm{~s}$

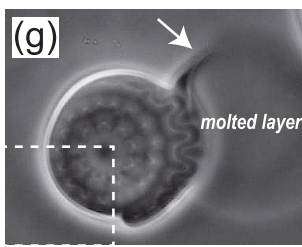

$t=1065 \mathrm{~s}$



$t=900 \mathrm{~s}$

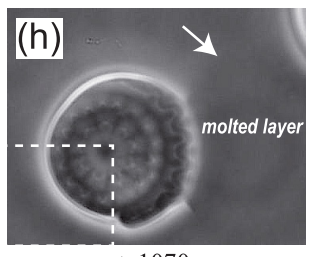

$t=1070 \mathrm{~s}$ (i)

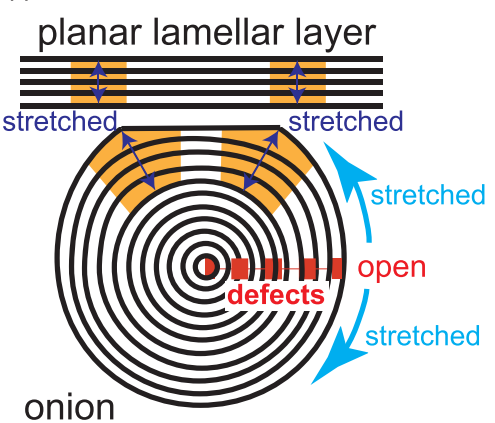

FIG. 4. [(a)-(h)] The same process as in Fig. 3, around onion 1 with a larger magnification and higher time resolution. The scale bar corresponds to $200 \mu \mathrm{m}$. We can see the development of a ring-like dotted pattern around the edge of the flattened region of the onion contacting with the planner lamellar layer. This pattern repeatedly appears with a temporal increase in the contact area. In [(e)-(h)], we indicate the center position of the onion in (e) by white dashed lines to show the slight relative motion of the core and molted parts of the onion [see Fig. 5(e). In [(f)-(h)], we also show the molted outer layer of the onion that merges with the planner lamellar layer by the white arrows [see also the regions surrounded by the white dashed curve in Fig. 3(f)]. (i) Schematic picture of an onion contacted to a planar lamellar layer. The flattening of a top part of the onion, i.e., the deviation from the spherical shape with the minimal surface area, causes lateral tension on the surface lamellar layer of the onion (see the light blue arrows). This tension acting on the surface layer of the onion may eventually open up the defects, leading to the pealing-off and detachment of the surface part. This surface layer molted from the onion wets to, spreads on, and then merges with the planar lamellar layer [see the arrowed parts in (f)-(h)]. We also note that the shape deformation of the onion described above leads to the mechanical tension along a radial direction in the onion around the edge of the contacted flattened region (see the orange regions and the dark blue arrows). We speculate that this mechanical stretching along the radial direction may cause the layer undulation instability under moderate stretching and the sponge droplet formation under intense stretching in the deformed ring-shaped deformed region of the onion (shaded in orange), leading to the wavy pattern and the ring-like dotted pattern, respectively. The instability periodically takes place when the accumulated stress becomes large enough to induce the instability.

curvature of the unperturbed spherical onion. Thus, this part of the lamellar structure in the onion and that of the planar lamellar layer [see the orange regions and the dark blue arrows in Fig. 4(i)] must be stretched perpendicular to the membranes. This effect is particularly significant in a ringlike region of the onion near the edge of the contacted region. This stretching along the radial direction should cause the instability in the ring-like contact line region suffering from large deformation [the orange region of the onion in Fig. 4(i)], whose mechanism is essentially the same as the layer-undulation instability of planar lamellar (smectic) order caused by stretching along layer-normal [1,51]. For keeping the phase coherence of the undulation in circular ring geometry, the frequency of the wavy pattern is quantized to an integer, which is reminiscent of the Bohr-Sommerfeld quantization condition in quantum mechanics. Since the undulation should be formed in the stretched direction, which is almost along the radial direction [see the dark blue allows in Fig. 4(i)], the wavy pattern should appear in the 2D projected image observed by optical microscopy. However, if the deformation is too large, the undulation may not be enough to compensate for the local decrease of the volume fraction induced by stretching. We speculate that the part of the lamellar phase may transform to the sponge phase, following the lever rule, and the resulting sponge droplets are formed in a ring-like white dotted pattern under the influence of undulation fluctuations.

The ring-like dotted pattern that appeared first has 9, 13, and 11 waves for onions $1-3$, respectively [see Fig. 3(b)], although the sizes of these onions are about the same [see Fig. 3(a)]. The number of dots seems to increase with the size of the contact area [dark areas in Fig. 3(a)], although this might be due to stochastic fluctuations.

The instability may occur each time when the accumulated mechanical stress overcomes the instability threshold. Thus, this instability takes place repeatedly, as observed in Figs. 4(e)-4(h). We can also see another interesting type of pattern, a wavy pattern, outside the ring-like dotted patterns [yellow arrows in Figs. 3(e) and 4(f)]. We speculate that the wavy pattern is also caused by the layer undulation instability mentioned above. The deformation seems large enough to induce the layer undulation instability but not enough to produce the sponge phase. A possible reason is that the outer layer already has opened up a hole around the linear array of defects, which relaxes the mechanical tension stretching the lamellar layer along the layer-normal direction [see the dark blue arrows in Fig. 4(i)]. These scenarios look reasonable but highly speculative. Further study is necessary to elucidate the valid mechanisms. Three-dimensional confocal observation may be helpful for this purpose.

\section{Summary of the coalescence process of onions and the flat lamellar layer}

Here, we summarize the coalescence process of onions and the flat lamellar layer formed on the glass wall. First, heterogeneous nucleation of the lamellar phase occurs on the glass wall (top), as shown in Fig. 5(a). This is because 
(a)

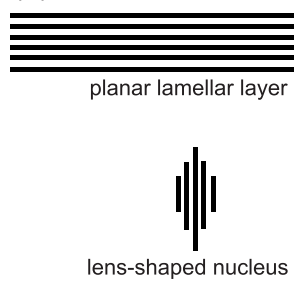

(d) (b)

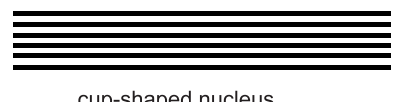

-shaped nucleus



(e)

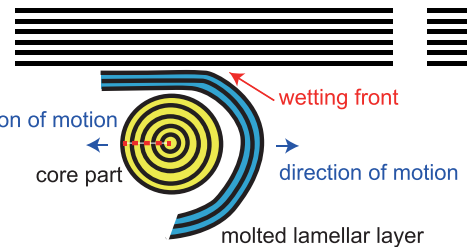

(c)

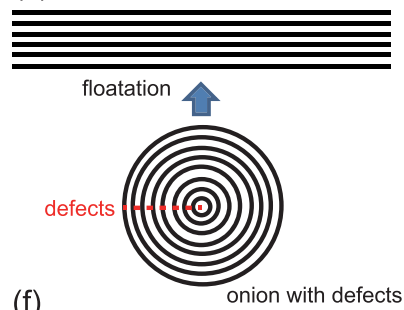

(f)

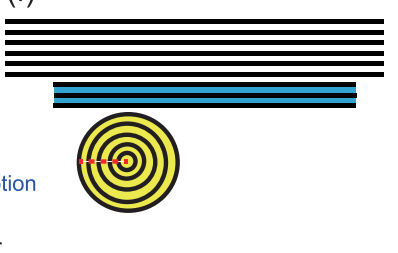

(g)

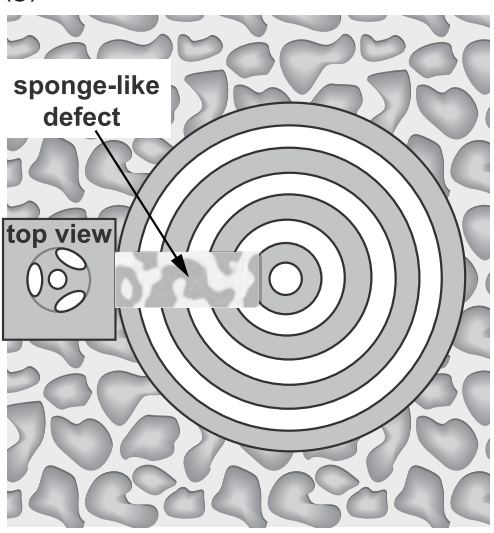

FIG. 5. Schematic figure explaining the coalescence process between an onion and a flat lamella. (a) Formation of the flat lamellar phase on the top glass wall, followed by homogeneous nucleation of a lamellar nucleus in bulk. (b) Spontaneous bending of the lamellar nucleus. (c) Onion formation. (d) Contact of the onion to the flat lamellar phase and the resulting onion deformation. Stress accumulation in the onion's orange part induces the separation between the surface (blue) and core (yellow) parts. (e) Molting of the surface part (blue) of the onion from the core part (yellow). In this process, the core part of the onion and the surface part move in the opposite direction [see the slight motion of the onion center in Figs. 4(e)-4(h)] (see also Movie S1 in the Supplemental Material [56]). (f) Spreading of the surface onion layer on the flat lamellar layer [see the regions surrounded by the white dashed curve in Figs. 3(e) and 3(f) and the regions pointed by the white arrow in Figs. 4(f)-4(h)]. The whole process [(a)-(f)] can be seen in Movie S1 in the Supplemental Material [56]. (g) Schematic figure of the onion with defects. Here, we do not show a detailed structure of defects and how membranes in onions are connected to those in the sponge-like defects due to the lack of microscopic information. What we can say at this stage is that the defects may have a sponge-like membrane configuration while keeping the inside-outside symmetry (without edges). The grey and white regions represent the inside and outside regions, respectively. We leave such clarification of the detailed defect structure for future investigation. On the possible structure of the sponge-lamellar interface, see e.g., Fig. 1 of Ref. [59].

wall-assisted heterogeneous nucleation costs lower interface energy than homogeneous nucleation in bulk. Later, the lamellar phase is spontaneously nucleated in bulk [Fig. 5(a)]. The lamellar nucleus has a nonspherical lens-like shape to keep an epitaxial relation between the lamellar and sponge phases at the interface (see Ref. [39] on the details of the shape transformation and the epitaxial relation in the same system; see also Refs. [57-62] and Fig. 1 of Ref. [59] on how membranes are connected across the interface between the lamellar and sponge phase). The flat lamellar configuration is beneficial in elastic energy but costs larger surface energy than the curved configuration. This surface energy penalty becomes bigger and bigger with the lamellar nucleus's growth, exceeding the bending energy cost. Therefore, the lamellar nucleus starts to bend as it grows. In this way, the lamellar nucleus becomes more and more spherical [see Fig. 5(b)] and eventually forms a spherical onion [Fig. 5(c)]. However, as is evident from the formation process, the onion structure is not seamless, contrary to the common belief; and it should have defects at the bonded part [Fig. 5(c)]. Thus, the structure of surfactant onions is not closed as real plant onions.

The key to understanding the phenomenon lies in the formation process of onions from the sponge phase. Since surfactant bilayer membranes have a seamless structure, i.e., the formation of edges is not allowed due to a too high free-energy cost, the sponge phase is separated into the inside and outside regions by membranes in a bicontinuous structure [49]. Such breaking of the inside-outside symmetry as a consequence of the absence of membrane edges was experimentally confirmed by perfect partitioning of colloidal particles into the two types of cells formed from the inside and outside water when the homogeneous sponge phase separates to a dense sponge and water phase [63]: Only one type of cell contains colloidal particles, whereas the other type of cell, separated from it by a single bilayer, is empty. Therefore, even after forming the onion phase, water regions sandwiched by membranes should still keep the inside and outside identity alternatively, as illustrated in Figs. 1(a) and 1(b). There is a considerable energy barrier for the reconnection of membranes involving topological changes. Thus, although such a reconnection may happen under strong external fields, spontaneous reconnection should be unlikely due to the high free-energy barrier, at least in a short time. This consideration strongly indicates the presence of an array of defects inside an onion, as shown in Fig. 1(b). Indeed, the existence of such defects will be evidenced by the process by which the onion and flat lamellar layers coalesce, as described below, although it is difficult to observe the defects directly. Since water permeability directly through the surfactant membrane is very low, the defective structure should play a pivotal role in exchanging water inside and outside the onion (see below).

Now, we consider a central question of how onions with defects merge the flat lamellar layer. When an onion touches the flat lamellar layer, first, its shape is deformed to a nonspherical shape with a flat part. This shape deformation induces two effects. The first effect is the layer-normal stretching of the lamellar structure in the part of the onion near the 
edge of the contact region [see Fig. 4(i) and 5(d)]. As explained above, the stretching of the lamellar layer along the layer-normal direction leads to the layer undulation instability [1,51]. This instability is caused by the physical constraint that the intermembrane spacing must be kept constant due to the conservation of the surfactant concentration (note that surfactant molecules are not transferred individually by diffusion in water because of a large free-energy penalty). If the deformation is too large, the lamellar order cannot be maintained in highly deformed regions and partially transforms to the sponge phase following the lever rule, forming tiny sponge droplets. Thus, the mechanical instability may be origins of the ring-like dotted and wavy patterns that appear in the onion contacted to the planar lamellar layer (see Figs. 3 and 4), as speculated above.

The second effect is the stretching of the outer layer of the onion. As the contact area increases with time by wetting, the surface area of the onion increases with time, and thus, the onion's surface layer part is more and more stretched [see the light-blue arrows in Fig. 4(i)]. When this stretching force is strong enough to open up the defect array in the onion, a hole is created in the outer part of the onion [the blue part of the onion in Fig. 5(d)]. This part is either a part of the onion but with a hole or separated from the core part that is much less deformed [see Fig. 5(e)]. Once detachment occurs, the sponge phase, isotropic fluid, flows into the opened part during this process. The thickness of the molted outer layer may be related to the penetration depth of the shape deformation [see the onion's blue skin part in Figs. 5(d) and 5(e)]. For reducing the surface energy and bending energy of the molted curved lamellar layer, this layer wets the flat lamellar layer, becomes flatter, and quickly spreads over it [see the white arrows in Figs. 4(f) and 4(g) and the wetting front indicated by the red arrow in Fig. 5(e)]. In this spreading process of the onion surface layer, the onion's core part slightly moves to the direction opposite to the outer layer spreading under the momentum conservation [Fig. 5(e)].

Then, this core part of the onion touches the lamellar layer again, repeating molting. Eventually, the onion is entirely absorbed into the flat lamellar layer formed on the top cover glass.

This opening of the onion surface layer by adhesion (i.e., wetting) force indicates that the onion structure is not a seamless closed structure with perfect spherical symmetry but a spherical structure with a line defect array [the red-dashed line in Fig. 1(b)]. We speculate that the onion defects may have a sponge-like membrane configuration while keeping the inside-outside symmetry (without edges), as schematically shown in Fig. 5(g). However, we leave such clarification of the defect structure for future investigation since we do not have any microscopic information on its details. Such onion defect formation is a natural consequence of the topological transition accompanying the Euler number's change.

\section{Additional pieces of evidence for presence of topological defects in onions}

Here, we show additional pieces of evidence for the presence of topological defects in the onion structure. One evidence is obtained by observing the reverse process of onion
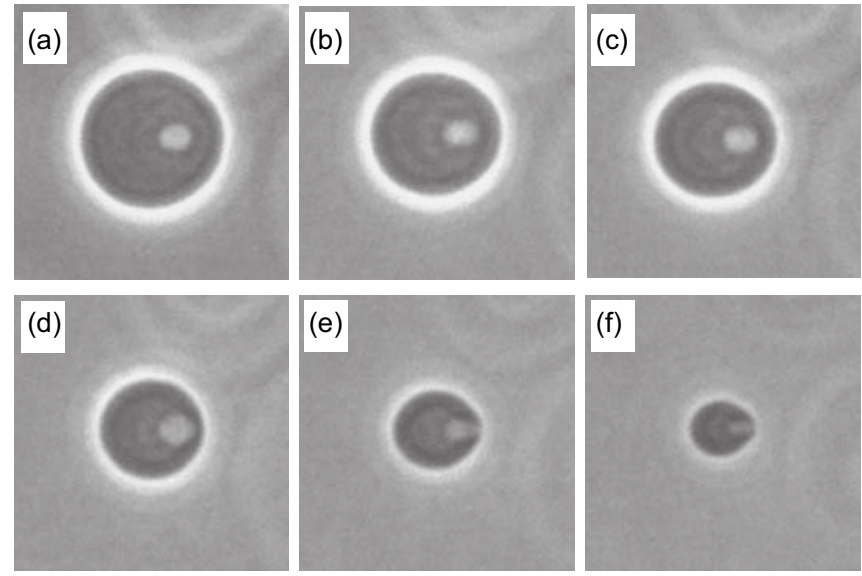

FIG. 6. The onion-to-sponge transformation process upon heating. The onion is first formed by cooling the sponge phase from $38.0^{\circ} \mathrm{C}$ to $36.0^{\circ} \mathrm{C}$ with the rate of $4 \mathrm{~K} / \mathrm{min}$ in the $5.0 \mathrm{wt} \%$ surfactant solution. After waiting for $5 \mathrm{~min}$, we heat the sample to $38.0^{\circ} \mathrm{C}$ with the same rate. The images [(a)-(f)] correspond to the structures at 1 , $1.3,1.6,1.9,2.2$, and $2.5 \mathrm{~s}$ after the temperature reaches $38.0^{\circ} \mathrm{C}$.

formation from the sponge phase, i.e., the onion-to-sponge transformation upon heating. The process is shown in Fig. 6 . We can see the onion's lamellar order melts from both the surface and center [see (a)-(c)]. The central sponge region changes its shape from a spherical to a tear-drop shape in the late stage, reflecting the topological defects. The melting process is the reverse process of onion formation [see Fig. 2(a)]. This observation also supports the absence of the spherical symmetry and the presence of the topological defect array.

Another evidence is observed in our experiments in which the temperature of an onion-sponge coexisting system is increased much more slowly compared to the above case. This temperature increase leads to a gradual decrease in the onion volume [see Fig. 7(b)], following the lever rule [see Fig, 1(c)], since the excess water is gradually expelled from the inside of onions to the sponge matrix phase. Here we note that the heating rate of $0.01 \mathrm{~K} / \mathrm{min}$ is slow enough for water's exchange inside and outside the onion. This observation indicates the presence of a channel for water exchange inside the onion structure. This channel should be the onion's internal defects [see the red dashed line in Fig. 1(b)]. Here we stress that water permeation through a membrane is negligible in the time scale of the heating process. It is supported by our previous measurements that the volume change of two water compartments separated by a single bilayer membrane (see Ref. [63] on the situation) was not detected over hours.

We also observe an interesting instability of the onion structure. The volume shrinking and the resulting decrease in the onion radius cause an excess of membrane surface areas inside the onion since the number of membranes is kept constant during this process. This excess area induces layer undulation instability and enhances corrugation amplitude, decreasing the corrugation frequency. For example, in Fig. 7, the corrugation frequency decreases from 8 to 5 in a discrete manner step by step. One might think that the decrease of the onion radius upon heating results from the onion being peeled. However, if this were the case, there would not appear 

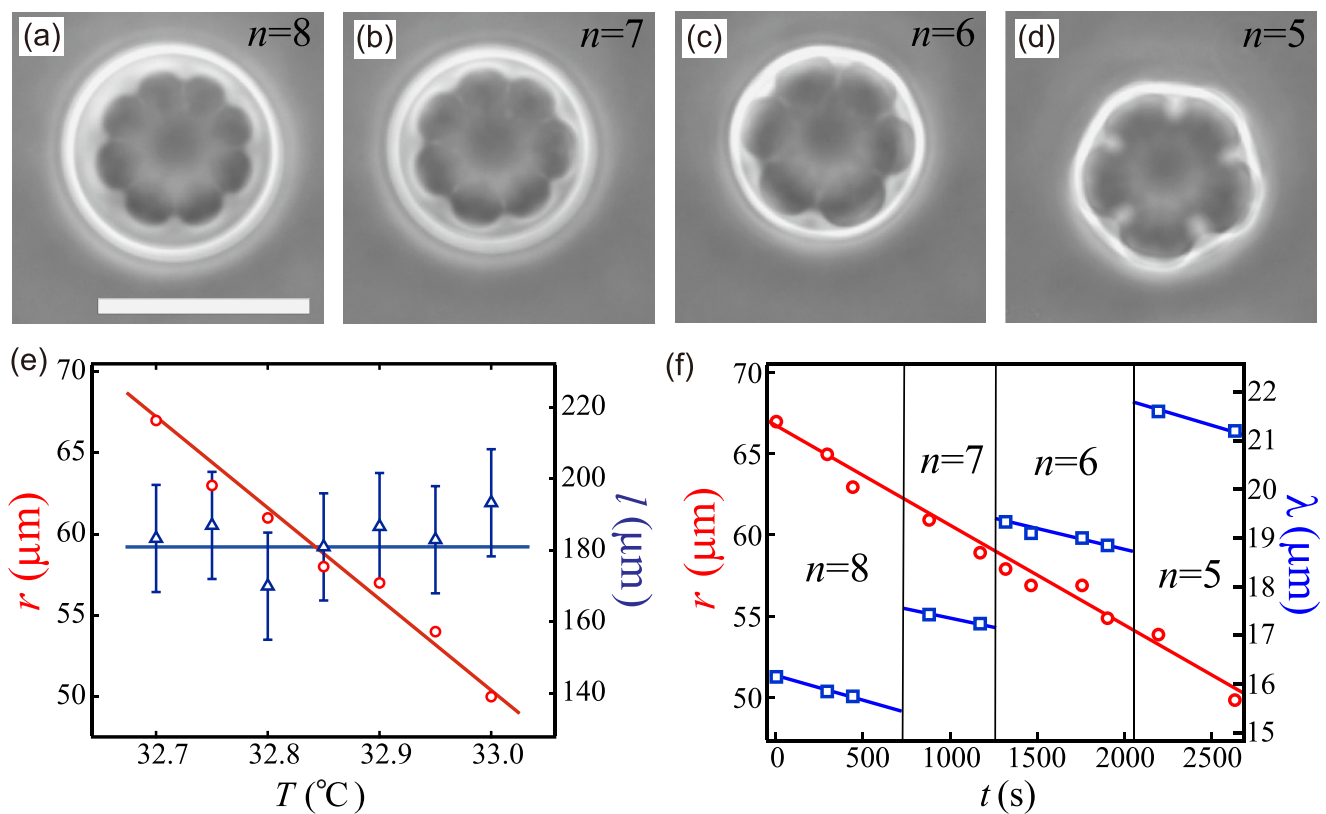

FIG. 7. Layer undulation instability of the lamellar structure in onions. Here $\phi=10.0 \mathrm{wt} \%$. [(a)-(d)] Development of undulation instability in an onion formed by a quench from $33.7^{\circ} \mathrm{C}$ to $32.7{ }^{\circ} \mathrm{C}$ with a cooling rate of $10 \mathrm{~K} / \mathrm{min}$, when we heat it with a rate of $0.01 \mathrm{~K} / \mathrm{min}(\mathrm{see}$ Movie $\mathrm{S} 2$ in the Supplemental Material [56] for the dynamic process, in which the acceleration factor is $\times 300)$. Images [(a)-(d)] correspond to those at (a) $32.7^{\circ} \mathrm{C}(t=300 \mathrm{~s})$, (b) $32.8^{\circ} \mathrm{C}(t=800 \mathrm{~s})$, (c) $32.9{ }^{\circ} \mathrm{C}(t=1200 \mathrm{~s})$, and (d) $33.0^{\circ} \mathrm{C}(t=2250 \mathrm{~s})$, where we set $t=0 \mathrm{~s}$ when starting to heat. We also show the number of corrugation $n$ in each panel. The scale bars correspond to $200 \mu \mathrm{m}$ for all. (e) Temperature dependence of the onion radius $r$ and the perimeter length of the dark corrugated central region $\ell$. The solid lines are eye guide. (f) Temporal change in the onion radius $r$ and the period of corrugation $\lambda$ during the slow heating process. We can see a discontinuous change in $\lambda$ : The number of corrugation decreases from $n=8$ to $n=5$ step by step.

the corrugation. The corrugation phenomenon can be regarded as evidence of the conservation of the number of stacked membranes in the onion. One might also think that individual surfactant molecules may diffuse in water to reorganize membrane configurations. Here we stress that the reorganization of surfactant membranes should be made while keeping membrane organization and not through the diffusion of individual surfactant molecules. It is because being individual surfactants in water involves a large free energy penalty. In contrast, in principle, water can be transferred from onions to the sponge phase through the defects in onions. This scenario may be only a plausible physical explanation derived from our optical microscopy observation, although we do not have microscopic support yet.

\section{CONCLUSION}

Finally, we discuss the implications of our findings. As discussed above, the fact that surfactant onions formed from the sponge phase are not seamless and have a group of defects on the axis passing the center is a consequence of the topological constraint coming from the energetic penalty for membrane edge formation. This onion formation process is markedly different from block copolymers, in which multilamellar structures are formed by structural ordering from a disordered melt state. In this case, onions may have defect- free closed structures. Carbon onion formation under electron irradiation may be similar to the block-copolymer case, although onions can also be formed through curling few-layer graphene flakes in the arc discharge synthetic process [64]. Thus, we may conclude that the topological character of onion structures is determined by the formation pathway, for example, whether it involves the sponge-to-lamellar transition or not.

Our finding would shed fresh light on the topological character of surfactant onion structures. It may also hint at a long-standing question on how onions are formed under shear from the planar or leak-like lamellar structures. Whether an onion structure is closed or not drastically influences the material exchange between its inside and outside, and thus, it should potentially impact the applications.

\section{ACKNOWLEDGMENTS}

We thank an anonymous referee for providing valuable comments. This work was partially supported by Grants-inAid for Scientific Research (A) (No. JP18H03675), Scientific Research (B) (No. JP20H01874), and Specially Promoted Research (No. JP25000002 and No. JP20H05619) from the Japan Society for the Promotion of Science (JSPS). We are also grateful for UROP (Undergraduate Research Opportunity Program) of the Institute of Industrial Science, the University of Tokyo. 
[1] M. Kleman and O. D. Lavrentovich, Soft Matter Physics: An Introduction (Springer, New York, 2003).

[2] G. Schmitz and G. Müller, Structure and function of lamellar bodies, lipid-protein complexes involved in storage and secretion of cellular lipids. J. Lipid Res. 32, 1539 (1991).

[3] V. Sørensen, A. Brech, D. Khnykin, E. Kolpakova, L. Citores, and S. Olsnes, Deletion mutant of FGFR4 induces onion-like membrane structures in the nucleus, J. Cell Sci. 117, 1807 (2004).

[4] P. Paumard, J. Vaillier, B. Coulary, J. Schaeffer, V. Soubannier, D. M. Mueller, D. Brèthes, J.-P. di Rago, and J. Velours, The ATP synthase is involved in generating mitochondrial cristae morphology, EMBO J. 21, 221 (2002).

[5] C. A. Mannella, Structure and dynamics of the mitochondrial inner membrane cristae, Biochim. Biophys. Acta. Mol. Cell Res. 1763, 542 (2006).

[6] D. Tarasenko, M. Barbot, D. C. Jans, B. Kroppen, B. Sadowski, G. Heim, W. Möbius, S. Jakobs, and M. Meinecke, The MICOS component Mic60 displays a conserved membrane-bending activity that is necessary for normal cristae morphology, J. Cell Biol. 216, 889 (2017).

[7] L. D. Mayer, M. J. Hope, P. R. Cullis, and A. S. Janoff, Solute distributions and trapping efficiencies observed in freeze-thawed multilamellar vesicles, Biochim. Biophys. Acta Biomembr. 817, 193 (1985).

[8] J. J. Moon, H. Suh, A. Bershteyn, M. T. Stephan, H. Liu, B. Huang, M. Sohail, S. Luo, S. H. Um, H. Khant et al., Interbilayer-crosslinked multilamellar vesicles as synthetic vaccines for potent humoral and cellular immune responses, Nat. Mater. 10, 243 (2011).

[9] C. Faure, A. Derré, and W. Neri, Spontaneous formation of silver nanoparticles in multilamellar vesicles, J. Phys. Chem. B 107, 4738 (2003).

[10] O. Regev, R. Backov, and C. Faure, Gold nanoparticles spontaneously generated in onion-type multilamellar vesicles. Bilayers-particle coupling imaged by cryo-TEM, Chem. Mater. 16, 5280 (2004).

[11] H. W. Kroto, Carbon allotropes - Carbon onions introduce new flavor to fullerene studies, Nature (London) 359, 670 (1992).

[12] D. Ugarte, Curling and closure of graphitic networks under electron-beam irradiation, Nature (London) 359, 707 (1992).

[13] V. L. Kuznetsov, I. L. Zilberberg, Y. V. Butenko, A. L. Chuvilin, and B. Segall, Theoretical study of the formation of closed curved graphite-like structures during annealing of diamond surface, J. Appl. Phys. 86, 863 (1999).

[14] F. B. Rosevear, The microscopy of the liquid crystalline neat and middle phases of soaps and synthetic detergents, J. Am. Oil Chem. Soc. 31, 628 (1954).

[15] A. D. Bangham and R. W. Horne, Negative staining of phospholipids and their structural modification by surface-active agents as observed in the electron microscope, J. Mol. Biol. 8, 660 (1964).

[16] A. D. Bangham, M. M. Standish, and J. C. Watkins, Diffusion of univalent ions across the lamellae of swollen phospholipids, J. Mol. Biol. 13, 238 (1965).

[17] D. D. Lasic, The mechanism of vesicle formation, Biochem. J. 256, 1 (1988).
[18] O. Diat and D. Roux, Preparation of monodisperse multilayer vesicles of controlled size and high encapsulation ratio, J. Phys. II (Paris) 3, 9 (1993).

[19] J. Zipfel, F. Nettesheim, P. Lindner, T. D. Le, U. Olsson, and W. Richtering, Cylindrical intermediates in a shear-induced lamellar-to-vesicle transition, Europhys. Lett. 53, 335 (2001).

[20] H. Miyazawa and H. Tanaka, Nucleation of lamellar domains from a sponge phase under shear flow: Shape selection of nuclei in a nonequilibrium steady state, Phys. Rev. E 76, 011513 (2007).

[21] D. Bonn, J. Meunier, O. Greffier, A. Al-Kahwaji, and H. Kellay, Bistability in non-Newtonian flow: Rheology of lyotropic liquid crystals, Phys. Rev. E 58, 2115 (1998).

[22] G. Schmidt, S. Müller, C. Schmidt, and W. Richtering, Rheooptical investigations of lyotropic mesophases of polymeric surfactants, Rheol. Acta 38, 486 (1999).

[23] A. Léon, D. Bonn, J. Meunier, A. Al-Kahwaji, O. Greffier, and H. Kellay, Coupling Between Flow and Structure for a Lamellar Surfactant Phase, Phys. Rev. Lett. 84, 1335 (2000).

[24] W. Richtering, Rheology and shear induced structures in surfactant solutions, Curr. Opin. Colloid Interface Sci. 6, 446 (2001).

[25] T. D. Le, U. Olsson, K. Mortensen, J. Zipfel, and W. Richtering, Nonionic amphiphilic bilayer structures under shear, Langmuir 17, 999 (2001).

[26] L. Courbin, J. P. Delville, J. Rouch, and P. Panizza, Instability of a Lamellar Phase Under Shear Flow: Formation of Multilamellar Vesicles, Phys. Rev. Lett. 89, 148305 (2002).

[27] H. Tanaka, M. Isobe, and H. Miyazawa, Shear-induced discontinuous and continuous topological transitions in a hyperswollen membrane system, Phys. Rev. E 73, 021503 (2006).

[28] B. Medronho, S. Shafaei, R. Szopko, M. G. Miguel, U. Olsson, and C. Schmidt, Shear-induced transitions between a planar lamellar phase and multilamellar vesicles: Continuous versus discontinuous transformation, Langmuir 24, 6480 (2008).

[29] L. Gentile, M. A. Behrens, L. Porcar, P. Butler, N. J. Wagner, and U. Olsson, Multilamellar vesicle formation from a planar lamellar phase under shear flow, Langmuir 30, 8316 (2014).

[30] S. Fujii and Y. Yamamoto, Dynamic orientation transition of the lyotropic lamellar phase at high shear rates, Soft matter 11, 9330 (2015).

[31] T. Kato, Shear-induced lamellar/onion transition in surfactant systems, in Advances in Biomembranes and Lipid SelfAssembly, Vol. 27 (Elsevier, Amsterdam, 2018), pp. 187-222.

[32] E. van der Linden, W. T. Hogervorst, and H. N. W. Lekkerkerker, Relation between the size of lamellar droplets in onion phases and their effective surface tension, Langmuir 12, 3127 (1996).

[33] A. G. Zilman and R. Granek, Undulation instability of lamellar phases under shear: A mechanism for onion formation? Eur. Phys. J. B 11, 593 (1999).

[34] G. K. Auernhammer, H. R. Brand, and H. Pleiner, Shearinduced instabilities in layered liquids, Phys. Rev. E 66, 061707 (2002).

[35] S. M. Fielding, Complex dynamics of shear banded flows, Soft Matter 3, 1262 (2007).

[36] P. D. Olmsted, Perspectives on shear banding in complex fluids, Rheol. Acta 47, 283 (2008) 
[37] H. Shiba, H. Noguchi, and G. Gompper, Structure formation of surfactant membranes under shear flow, J. Chem. Phys. 139, 014702 (2013).

[38] L. Courbin, G. Cristobal, J. Rouch, and P. Panizza, Closed compact Taylor's droplets in a phase-separated lamellarsponge mixture under shear flow, Europhys. Lett. 55, 880 (2001).

[39] Y. Iwashita and H. Tanaka, Spontaneous Onion-Structure Formation From Planar Lamellar Nuclei, Phys. Rev. Lett. 98, 145703 (2007).

[40] Y. Dou, P. Long, S. Dong, and J. Hao, Spontaneous transformation of lamellar structures from simple to more complex states, Langmuir 29, 12901 (2013).

[41] S. Khodaparast, W. Sharratt, H. Wang, E. S. J. Robles, R. Dalgliesh, and J. T. Cabral, Spontaneous formation of multilamellar vesicles from aqueous micellar solutions of sodium linear alkylbenzene sulfonate (NaLAS), J. Colloid Interface Sci. 546, 221 (2019).

[42] S. Koizumi, H. Hasegawa, and T. Hashimoto, Ordered structures of block copolymer/homopolymer mixtures. 5. Interplay of macro- and microphase transitions, Macromolecules 27, 6532 (1994).

[43] L. Li, K. Matsunaga, J. Zhu, T. Higuchi, H. Yabu, M. Shimomura, H. Jinnai, R. C. Hayward, and T. P. Russell, Solvent-driven evolution of block copolymer morphology under 3D confinement, Macromolecules 43, 7807 (2010).

[44] H. Shen and A. Eisenberg, Control of architecture in blockcopolymer vesicles, Angew. Chem. Int. Ed. Engl. 39, 3310 (2000).

[45] J. M. Shin, Y. Kim, H. Yun, G.-R. Yi, and B. J. Kim, Morphological evolution of block copolymer particles: Effect of solvent evaporation rate on particle shape and morphology, ACS Nano 11, 2133 (2017).

[46] E. Avalos, T. Teramoto, H. Komiyama, H. Yabu, and Y. Nishiura, Transformation of block copolymer nanoparticles from ellipsoids with striped lamellae into onionlike spheres and dynamical control via coupled Cahn-Hilliard equations, ACS Omega 3, 1304 (2018).

[47] S. Zhang, H.-J. Sun, A. D. Hughes, R.-O. Moussodia, A. Bertin, Y. Chen, D. J. Pochan, P. A. Heiney, M. L. Klein, and V. Percec, Self-assembly of amphiphilic janus dendrimers into uniform onion-like dendrimersomes with predictable size and number of bilayers, Proc. Natl. Acad. Sci. USA 111, 9058 (2014).

[48] S. E. Wilner, Q. Xiao, Z. T. Graber, S. E. Sherman, V. Percec, and T. Baumgart, Dendrimersomes exhibit lamellar-to-sponge phase transitions, Langmuir 34, 5527 (2018).

[49] D. Roux, C Coulon, and M. E. Cates, Sponge phases in surfactant solutions, J. Phys. Chem. 96, 4174 (1992).
[50] Y. Iwashita and H. Tanaka, Self-organization in phase separation of a lyotropic liquid crystal into cellular, network and droplet morphologies, Nat. Mater. 5, 147 (2006).

[51] W. Helfrich, Steric interaction of fluid membranes in multilayer systems, Z. Natureforsch. 33a, 305 (1978).

[52] Y. Iwashita and H. Tanaka, Surface-Assisted Monodomain Formation of an Ordered Phase of Soft Matter Via the First-Order Phase Transition, Phys. Rev. Lett. 95, 047801 (2005).

[53] J. Yamamoto and H. Tanaka, Shear-Induced Sponge-toLamellar Transition in a Hyperswollen Lyotropic System, Phys. Rev. Lett. 77, 4390 (1996).

[54] S. T. Milner, M. E. Cates, and D. Roux, Hydrodynamic modes and topology in microemulsions and 13 phases, J. Phys. 51, 2629 (1990).

[55] G. Porte, M. Delsanti, I. Billard, M. Skouri, J. Appell, J. Marignan, and F. Debeauvais, Scaling laws for some physical properties of the 13 (sponge) phase, J. Phys. II France 1, 1101 (1991).

[56] See Supplemental Material at http://link.aps.org/supplemental/ 10.1103/PhysRevResearch.3.043094 for Supplemental Movies (Movie S1 corresponding to Fig. 3 and Movie S2 corresponding to Figs. 7(a)-7(d); the acceleration factor is $\times 150$ for Movie $S 1$ and $\times 300$ for Movie S2).

[57] C. Quilliet, C. Blanc, and M. Kleman, Evidence of a Phenomenon of Epitaxy at the Interface Between a Lamellar $\mathrm{L}_{\alpha}$ Phase and $\mathrm{a} \mathrm{L}_{3}$ Sponge, Phys. Rev. Lett, 77, 522 (1996).

[58] O. D. Lavrentovich, C. Quilliet, and M. Kleman, Orientational dependence of the surface free energy of the $\mathrm{L}_{\alpha}-\mathrm{L}_{3}$ interface, J. Phys. Chem. B 101, 420 (1997).

[59] C. Blanc, O. Sanseau, and V. Cabuil, The interface between the lamellar and the sponge phases, Mol. Cryst. Liquid Cryst. Sci. Tech. A. 332, 523 (1999).

[60] M. Buchanan, L. Starrs, S. U. Egelhaaf, and M. E. Cates, Kinetic pathways of multiphase surfactant systems, Phys. Rev. E 62, 6895 (2000).

[61] C. Blanc, Measurement of the anisotropy of the energy of an isotropic-smectic interface in a smectic cylinder: Application to the $\mathrm{L}_{3}-\mathrm{L}_{\alpha}$ interface, Phys. Rev. E 64, 011702 (2001).

[62] C. Blanc, Estimation of the interfacial tension between the lamellar and the sponge phases of a lyotropic system, Soft Mater. 1, 63 (2002).

[63] H. Tanaka, M. Isobe, and J. Yamamoto, Spontaneous Partitioning of Particles into Cellar Structures in a Membrane System, Phys. Rev. Lett. 89, 168303 (2002).

[64] M. Li, W. Liu, H. Zhang, Z. Liang, P. Duan, X. Yan, P. Guan, B. $\mathrm{Xu}$, and J. Guo, Direct imaging of construction of carbon onions by curling few-layer graphene flakes, Phys. Chem. Chem. Phys. 20, 2022 (2018). 